\title{
Automatic method of the technical state changes model generation
}

\author{
Michat Pająk ${ }^{1^{*}}$ \\ ${ }^{1}$ Faculty of Mechanical Engineering, University of Technology and Humanities in Radom, \\ Stasieckiego 54, 26-600 Radom, Poland
}

\begin{abstract}
One of the main objectives of technical diagnostics is identification the technical state of the machine. The technical state is described by the values of the object cardinal features. Unfortunately, during the operation process, some of the values of these features cannot be measured. It is proposed to estimate the values of the cardinal features based on their initial values and the influence of the forcing factors described by the time histories of the parameters of the operation process. Due to the limited knowledge of the correlation between operational parameters and forcing factors it was modelled in form of fuzzy Mamdani models. In the paper universal automatic method of the models generation was presented. Thanks to it, it was possible to generate the model of the technical state changes in automatic manner. The results of the usage of the generated model in case of real complex technical system can be found at the end of the paper.
\end{abstract}

\section{Introduction}

The subject of the considerations is a complex, critical technical system of strategic importance. Its technical condition is defined by the values of its cardinal features. However, it is not always possible to measure these values in real time. Changes in the values of the cardinal features of the system occur during the execution of the operational processes as a result of the influence of forcing factors. The level of the forcing factors' impact is determined by the manner of execution of the operational process and the intensity of the environmental impact on the system. If the intensity of the environmental impact on the system is assumed to be an independent value, the operational process is described only with the values of the parameters at which it takes place. The intensity of this process is therefore determined by the time histories of its parameters.

To model changes in the technical condition of the system, a model was proposed which, based on the time histories of the operational parameters, will estimate the levels of the impact of forcing factors occurring during the system operation. Then, on the basis of the levels of influence of forcing factors, changes in the values of cardinal features of the system will be identified. Finally, on the basis of changes in the value of the cardinal features of the system, a change in the its technical condition will be determined. The application of the

\footnotetext{
*Corresponding author: m.pajak@uthrad.pl
} 
developed model will be used to determine the technical condition of the system at time $t_{2}$ on the basis of the technical condition of the system at time $t_{l}$ and the time histories of the operational parameters in the time interval $\left[t_{1}, t_{2}\right](1)$.

$$
\left(s\left(t_{1}\right), p o_{1}(t)\left|\begin{array}{l}
t_{2} \\
t_{1}
\end{array}, \cdots, p o_{\text {npo }}(t)\right| \begin{array}{l}
t_{2} \\
t_{1}
\end{array}\right) \stackrel{M \Delta S}{\longrightarrow} s\left(t_{2}\right)
$$

where: $s(t)$ - the technical state of the system for time $t, p o_{i}(t) \mid t_{2}$ - time history of $i$-ts operational parameter in the time interval $\left[t_{1}, t_{2}\right], M \Delta S$ - the model of the technical state changes.

For the model, the input parameters are the technical state of the system at the initial moment of the operation $t_{l}$ and the time histories of the operational parameters. The initial technical state of the system is approximated as a result of the dependence on the course of the production phase and the course of pre-use processes of the machine. Similarly, the values of the operational parameters may belong to the group of measurable or non-measurable values. Thus, these values can only be approximated. Therefore, the created model of the process of changes in the technical condition must be designed in such a way as to take into account the approximate nature of the input data. Therefore, it was assumed that it will be developed using fuzzy modelling in the form of a set of fuzzy models where each transforms the time histories of the operational parameters into a change in the value of a specified cardinal feature of the system.

\section{The procedure of the model generation}

The task of creation models of the changes of the values as a function of time histories of the system operational parameters was solved according to the method of the Mamdani fuzzy models automatic generation based on MISO sample data [1,2]. Data samples, were prepared in a form of reduced measurement vectors (2).

$$
r m v_{j}\left(x_{l}\right):\left[\delta p o_{1}, \delta p o_{2}, \cdots, \delta p o_{n i p o}, \Delta x_{l}\right]
$$

where : $x_{l}$-the $l$-th cardinal feature, $p o_{i}-$ the $i$-th operational parameter $i=1, \ldots, n i p o\left(x_{l}\right)$, nipo $\left(x_{l}\right)$ - the amount of the significant operational parameters in term of system feature $x_{l}$, $r m v_{j}$ - the $j$-th reduced measurement vector, $\delta p o_{i}$ - the distance between time history of $i$-th operational parameter and its zeros time history (the time history of the specified operational parameter for minimum change of the feature value), $\Delta x_{l}$ - the change of the $l$-th system feature value.

Used method of automatic fuzzy models generation consist of three stages: generation, simplification and tuning. Generation is an iteration process that makes a predefined number of genetic algorithm cycles in every step. The result of this algorithm is a rule of fuzzy model knowledgebase [3]. Every generated rule allows to determine the degree of coverage the measuring samples by the fuzzy model. Samples with the coverage degree greater than or equals to the defined one are deleted. The algorithm is processed until all the existing measurement vectors are excluded. At the moment when the process of model generation in the particular step creates the rule with the coverage degree equal 0 this rule is rejected and probability of mutation of the genetic algorithm of the generation process increase according to formula (3)

$$
P_{m}^{\prime}=P_{m}+\frac{\left(1-P_{m}\right)}{\text { nuis }}
$$


where: $P_{m}$ - probability of mutation, $P^{\prime}{ }_{m}$ - modified probability of mutation, nuis - specified number of allowed iteration steps without the result.

In the moment when algorithm creates the rule with the coverage degree different then 0 the probability of mutation is back to its initial value and the process goes on.

Genetic algorithm is applied to the population where every chromosome represents single rule. Chromosomes are analysed for specified fitness function. In order to increasing the quality of the operation of the algorithm, evolution strategy is applied as a tool of local tuning of generated rules [4].

Each linguistic value of model input and output parameters is described by five characteristic values $r l, a, b, c, r r$, which meaning depends on the type of fuzzy set used for description the specified linguistic value. $r r$ and $r l$ are right and left range of the domain of input or output model parameters and they are equal for all the linguistic values of that parameter while interpretation of $a, b, c$ characteristic values for the specific types of fuzzy sets differs and is presented is the figures (Fig.1, Fig. 2, Fig. 3)

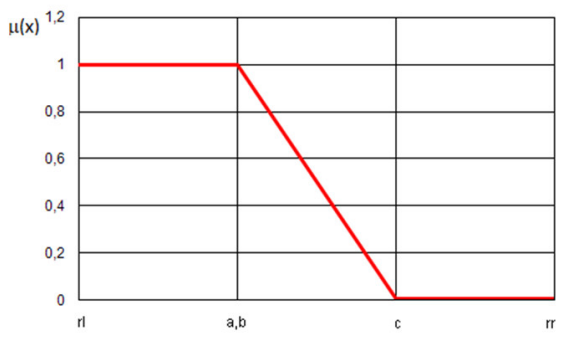

Fig. 1 - The interpretation of the characteristic values for fuzzy set type $L$ where $\mu(x)$ - values of the membership function

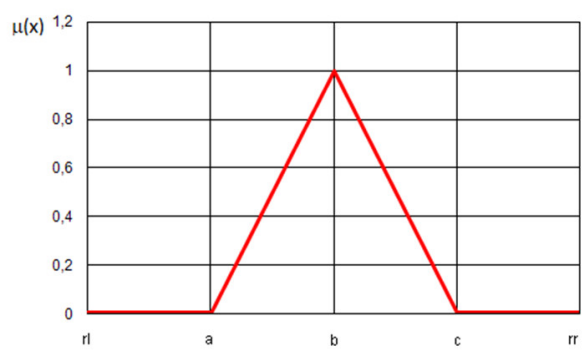

Fig. 2 - The interpretation of the characteristic values for fuzzy set type $\Lambda$ where $\mu(x)$ - values of the membership function

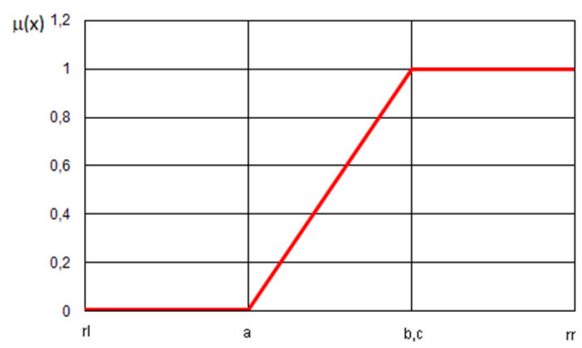

Fig.3 - The interpretation of the characteristic values for fuzzy set type $\Gamma$ where $\mu(x)$ - values of the membership function 
Each of the values $a, b, c$ has defined range of values (4), (5), (6).

$$
\begin{aligned}
& a \in\left[a-\frac{b-a}{2}, a+\frac{b-a}{2}\right] \\
& b \in\left[b-\frac{b-a}{2}, b+\frac{c-b}{2}\right] \\
& c \in\left[c-\frac{c-b}{2}, c+\frac{c-b}{2}\right]
\end{aligned}
$$

where: $a, b, c$-the characteristic values of the linguistic values.

In the generation process the chromosomes under genetic process are coded by means of real numbers. Using them the fuzzy model knowledgebase is coded. Each chromosome consists of two parts $C_{i}{ }^{l}$ and $C_{i}^{2}(7)$

$$
C_{i}=C_{i}^{1} C_{i}^{2}
$$

where: $C_{i}$ - the chromosome of genetic algorithm of the fuzzy model generating process, $C_{i}^{1}$ - first part of a chromosome, $C_{i}^{2}$ - second part of a chromosome.

The first part $C_{i}{ }^{l}$ codes linguistic values of model inputs and output whereas the second one $C_{i}^{2}$ codes values $a, b, c$ of every linguistic value from the first part (8), (9).

$$
C_{i}^{1}=\left(L_{\delta p o_{1}}^{j}, L_{\delta p o_{2}}^{k}, \ldots \ldots . ., L_{\delta p o_{\text {nipo }}}^{m}, L_{\Delta x_{F M}}^{n}\right)
$$

where: $C_{i}^{1}$ - first part of chromosome of genetic algorithm, $L_{\delta p o_{i}}^{j}-j$-th linguistic value of $i$-th input parameter of model, nipo - number of input parameters of fuzzy model of the change in system feature value, $L_{\Delta x_{F M}}^{i}-i$-th linguistic value of output parameter.

$$
\begin{aligned}
C_{i}^{2}= & \left(a\left(L_{\delta p o_{1}}^{j}\right), b\left(L_{\delta p o_{1}}^{j}\right), c\left(L_{\delta p o_{1}}^{j}\right),\right. \\
& a\left(L_{\delta p o_{2}}^{k}\right), b\left(L_{\delta p o_{2}}^{k}\right), c\left(L_{\delta p o_{2}}^{k}\right), \\
& \vdots \\
& a\left(L_{\delta p o_{\text {nipo }}}^{m}\right), b\left(L_{\delta p o_{n i p o}}^{m}\right), c\left(L_{\delta p o_{\text {nipo }}}^{m}\right), \\
& \left.a\left(L_{\Delta x_{F M}}^{j}\right), b\left(L_{\Delta x_{F M}}^{j}\right), c\left(L_{\Delta x_{F M}}^{j}\right)\right)
\end{aligned}
$$

where: $C_{i}^{2}$ - second part of chromosome of genetic algorithm, $L_{\delta p o_{i}}^{j}-j$-th linguistic value of $i$-th input parameter of model, nipo - number of input parameters of fuzzy model of the change in system feature value, $L_{\Delta x_{F M}}^{i}-i$-th linguistic value of output parameter, $a, b, c$ - characteristic values of input and output parameters of fuzzy model.

Domains for every gene of the first part of the chromosome are limited by value 1 and the amount of linguistic values defined for model parameters. For every gene of the second part of chromosome domains are defined according to formulas presented earlier (4), (5), (6).

The starting population of genetic algorithm consists of three equal parts. The first part consists of rules generated on the basis of measurement data, assuming values of the genes of the first part of the chromosome as linguistic values for which the value of the membership function is the maximum. The second part is made up of chromosomes with the same first part, while the second part is randomly generated. The third part of the pool is generated entirely randomly. The fitness function is given by the formula (10). The algorithm tends to maximize this function [5].

$$
Q(F M R)=T F(F M R) \cdot M S O(F M R) \cdot \operatorname{ICOF}(F M R) \cdot \operatorname{GIF}(F M R)
$$

where: $Q$ - the fitness function of the chromosome describing the FRM rule, FMR - fuzzy model inference rule, $T F$ - triggering frequency of the fuzzy model rule on the set of reduced measurement vectors, $M S O$ - average coverage coefficient of the set of reduced measurement vectors compliant with the fuzzy model rule, ICOF - coefficient of occurrence of reduced 
measurement vectors inconsistent with the fuzzy model rule, GIF - interaction coefficient of the fuzzy set rule with the rules already generated.

The individual parts of the fitness function are described by expressions (11) - (21) [6]:

$$
T F(F M R)=\frac{\sum_{i=1}^{n r m v} C F\left(F M R, w p_{i}\right)}{n r m v}
$$

where: $T F$ - triggering frequency of the fuzzy model rule on the set of reduced measurement vectors, $F M R$ - inference rule of the fuzzy model, $n r m v$ - number of reduced measurement vectors, $r m v_{i}-i$-th reduced measurement vector, $C F$ - coefficient of compliance of the fuzzy model rule and the reduced measurement vector

$$
\begin{aligned}
& C F(F M R, r m v)= \\
& \min \left(\mu_{L_{\delta p p_{1}}^{j}}\left(\delta p o_{1}\right), \ldots \ldots, \mu_{L_{\delta p_{n i p o}}^{m}}\left(\delta p e_{\text {nipo }}\right), \mu_{L_{\Delta_{\Delta_{F} M}}^{n}}\left(\Delta x_{F M}\right)\right)
\end{aligned}
$$

where: $\mu_{L_{\delta \rho_{1}}^{j}}$ - the value of the membership function of the $j$-th linguistic value of the input parameter, $\delta p o_{i}$ - crisp value of the $i$-th input parameter of the model, $\mu_{L_{\Delta_{x_{F} M}^{i}}}$ - the value of the membership function of $i$-th linguistic value of the output parameter, $\Delta x_{F M}$ - crisp value of the output parameter of the fuzzy model

$$
M S O(F M R)=\sum_{r m v \in S r m v_{+}(F M R)} \frac{C F(F M R, r m v)}{n r m v_{+}(F M R)}
$$

where: $M S O$ - the average coverage coefficient of the reduced measurement vectors set compliant with the fuzzy model rule, Srmv $v_{+}$- the set of reduced measurement vectors compliant with the fuzzy model rule

$$
\begin{gathered}
S r m v_{+}(F M R)=\left\{r m v \in \operatorname{Srmv}: C F(F M R, r m v) \geq \omega_{z}\right\} \\
n r m v_{+}(F M R)=\operatorname{card}\left(\operatorname{Srmv}_{+}(F M R)\right)
\end{gathered}
$$

where: $S r m v$ - set of reduced measurement vectors, $\omega_{z}$ - limit of compliance of the reduced measurement vector with the rule, $n r m v_{+}-$number of reduced measurement vectors compliant with the fuzzy model rule, card - power of the set

$$
\operatorname{ICOF}(F M R)=\left\{\begin{array}{l}
1, \\
n r m v_{-}(F M R) \leq k_{i c F M R} \cdot n r m v_{+}(F M R) \\
\frac{1}{n r m v_{-}(F M R)-k_{i c F M R} \cdot n r m v_{+}(F M R)+\exp (1)} \\
n r m v_{-}(F M R)>k_{i c F M R} \cdot n r m v_{+}(F M R)
\end{array},\right.
$$

where: ICOF - the coefficient of the occurrence of reduced measurement vectors inconsistent with the fuzzy model rule, $k_{i c F M R}$ - the ratio of the number of reduced measurement vectors inconsistent with the fuzzy model rule to the number of reduced measurement vectors compliant with the rule, values from the interval $[0,1], n r m v_{+}-$the number of reduced measurement vectors consistent with the fuzzy model rule, nrmv - the number of reduced measurement vectors inconsistent with the fuzzy model rule

$$
n r m v_{-}(F M R)=\operatorname{card}\left(\operatorname{Srm}_{-}(F M R)\right)
$$

where: $S r m v_{-}$- set of reduced measurement vectors inconsistent with the fuzzy model rule

$$
S r m v_{-}(F M R)=\{r m v \in S r m v: C F(F M R, r m v)=0 \wedge F L(F M R, r m v)>0\}
$$

where: $F L$ - the degree of fulfilment of the fuzzy model rule premises by the reduced measurement vector,

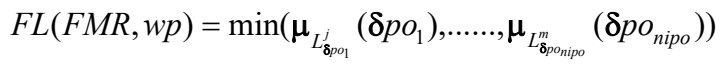




$$
\begin{aligned}
& \operatorname{GIF}(F M R)= \\
& 1-\max \left(C F\left(F M R, r m v\left(F M R_{1}\right)\right), \ldots, C F\left(F M R, r m v\left(F M R_{i}\right)\right)\right) \wedge \\
& i=1, \ldots \ldots ., n g r b r
\end{aligned}
$$

where: GIF - the interaction coefficient of fuzzy model rule with rules already generated, $n g r b r$ - the amount of the generated rules of the fuzzy model, $r m v\left(F M R_{i}\right)$ - inputs-output sample representing $i$-th inference rule of the fuzzy model

$$
\begin{aligned}
& r m v\left(F M R_{i}\right)= \\
& {\left[\begin{array}{lllll}
b\left(L_{\delta p o_{1}}^{j}\left(F M R_{i}\right)\right) & b\left(L_{\delta p o_{2}}^{k}\left(F M R_{i}\right)\right) & \ldots & b\left(L_{\delta p o_{\text {nipo }}}^{m}\left(F M R_{i}\right)\right) & b\left(L_{\Delta x_{F M}}^{n}\left(F M R_{i}\right)\right)
\end{array}\right]}
\end{aligned}
$$

where: $L_{\delta p o_{k}}^{j}\left(F M R_{i}\right)$ - the linguistic value for $k$-th input parameter of $i$-th rule, $b$-the characteristic value of the fuzzy set (Fig. 1, Fig. 2, Fig. 3).

A single cycle of the genetic algorithm involves three operations: crossover, mutation and an evolutionary strategy [7]. Crossover is performed with the help of different operators depending on the chromosomes involved in the operation. If the chromosomes have the same first parts, the arithmetic operator MIN-MAX [8] is used to cross the second parts. In this process, offspring are generated from the chromosomes to be crossed (22) according to the scheme (23) - (27).

$$
C_{1}=\left(c_{1}^{1}, c_{2}^{1}, \ldots \ldots ., c_{3 \cdot(n i p o+1)}^{1}\right), C_{2}=\left(c_{1}^{2}, c_{2}^{2}, \ldots \ldots . ., c_{3 \cdot(n i p o+1)}^{2}\right)
$$

where: $C_{1}, C_{2}$ - the chromosomes to be crossed, $c_{j}^{i}$ - the value of the $j$-th gene of $i$-th chromosome, nipo - the amount of the input parameters of the fuzzy model of the system feature change.

$$
\begin{gathered}
H_{k}=\left(h_{1}^{k}, h_{2}^{k}, \ldots \ldots . ., h_{3 \cdot(\text { nipo }+1)}^{k}\right) \wedge k=1,2,3,4 \\
h_{j}^{1}=\lambda_{g} \cdot c_{j}^{1}+\left(1-\lambda_{g}\right) c_{j}^{2} \\
h_{j}^{2}=\lambda_{g} \cdot c_{j}^{2}+\left(1-\lambda_{g}\right) c_{j}^{1} \\
h_{j}^{3}=\max \left(c_{j}^{1}, c_{j}^{2}\right) \\
h_{j}^{4}=\min \left(c_{j}^{1}, c_{j}^{2}\right)
\end{gathered}
$$

where: $H_{k}$ - the offspring chromosomes, $h_{j}^{k}$ - the value of the $j$-th gene of $k$-th offspring chromosome, $\lambda_{g}$ - genetic coefficient of crossover operator.

The two best offspring chromosomes take place of the parent chromosome in the pool that is subject to further optimization.

If the first parts of the parent chromosomes are different, a simple crossing is performed on the first parts and the values from the corresponding genes in the second part are copied. In this case, the first parts of the chromosomes are generated according to the scheme (28)

$$
H_{1}=\left(c_{1}^{1}, c_{2}^{1}, \ldots \ldots . c_{j}^{1}, c_{j+1}^{2}, \ldots \ldots, c_{3 \cdot(n i p o+1)}^{2}\right), H_{2}=\left(c_{1}^{2}, c_{2}^{2}, \ldots \ldots . c_{j}^{2}, c_{j+1}^{1}, \ldots \ldots, c_{3 \cdot(n i p o+1)}^{1}\right)
$$

where: $H_{1}, H_{2}$ - the offspring chromosomes, $c_{j}^{i}$ - the value of the $j$-th gene of $i$-th parent chromosome, nipo - the amount of the input parameters of the fuzzy model of the system feature change.

Two operators are also used in the mutation operation. If the selected mutation point is a gene in the second part of the chromosome, the non-uniform mutation operator [9] is used according to the formulas (29), (30)

$$
h_{j}^{i}= \begin{cases}c_{j}^{i}+\Delta\left(n g_{a c t}, r g_{\max }-c_{j}^{i}\right) & \text { dla } \tau_{g}=0 \\ c_{j}^{i}-\Delta\left(n g_{a c t}, c_{j}^{i}-r g_{\text {min }}\right) & \text { dla } \tau_{g}=1\end{cases}
$$


where: $h_{j}^{i}$ - the value of the $j$-th gene of $i$-th offspring chromosome, $c_{j}^{i}$ - the value of the $j$-th gene of $i$-th parent chromosome, $n g_{a c t}$ - the number of the actual generation, $r g_{\max }$ - the high range of the gene domain, $r g_{\min }$ - the low range of the gene domain, $\tau_{g}$ - the digit $\{0 ; 1\}$.

$$
\Delta\left(n g_{\text {act }}, y\right)=y\left(1-\gamma_{g}{ }^{\left(1-\frac{n g_{a c t}}{n g_{\max }}\right)^{\beta_{g}}}\right)
$$

where: $y$ - the input parameter of the function, $n g_{\max }$ - maximum generation number, $\tau_{g}$ - the digit $\{0 ; 1\}, \beta_{g}$-the parameter of non-uniform mutation, $\gamma_{g}$ - random value from range $[0,1]$.

However, if the gene selected for mutation is in the first part, its value is randomly decreased or increased by one. In case the operation is not possible due to the gene domain, the opposite operation is performed. Obviously, changing the value of the genes in the first part involves changing the values of corresponding genes in the second part of the chromosome.

The last operation of the genetic algorithm step is the evolution strategy [10]. It is applied to the specified part of the best chromosome. The operation modifies the values of the genes from the second part of the chromosome, determining their value according to formula (31)

$$
\begin{gathered}
h_{j}^{i}=c_{j}^{i}+\sigma_{j} \\
\sigma_{j \text { max }}=\sigma \cdot \frac{c_{j \text { max }}^{i}-c_{j \text { min }}^{i}}{4}
\end{gathered}
$$

where: $h_{j}^{i}$ - the value of the $j$-th gene of $i$-th offspring chromosome, $c_{j}^{i}$ - the value of the $j$-th gene of $i$-th parent chromosome, $\sigma_{j}$ - the value uniformly randomly generated from range $\left[0, \sigma_{\text {imax }}\right], \quad \sigma_{\text {jmax }}-$ the maximum value of the evolution strategy parameter, $c_{j \max }{ }^{i}-$ the maximum value of the $j$-th gene of $i$-th chromosome, $c_{j \min }{ }^{i}$ - the minimum value of the $j$-th gene of $i$-th chromosome, $\sigma$ - the parameter of the evolution strategy.

The value $\sigma$ is mutated according to formula (33)

$$
\sigma^{\prime}= \begin{cases}\frac{\sigma}{\sqrt[n m s e]{c_{s e}}} & \text { dla } p_{s e}>\frac{1}{5} \\ \sigma \cdot \sqrt[n m s e]{c_{s e}} & \text { dla } p_{s e}<\frac{1}{5} \\ \sigma & \text { dla } p_{s e}=\frac{1}{5}\end{cases}
$$

where: $\sigma$ - the parameter of the evolution strategy, $\sigma^{\prime}-$ the value of the parameter of the evolution strategy after mutation, $c_{s e}$ - the coefficient of the evolution strategy, nmse - the amount of the mutations of the evolution strategy, $p_{s e}-$ the relative frequency of the positive mutations of the evolution strategy.

If the modified chromosome is better according to the criterion contained in the fitness function, it replaces the parent chromosome, otherwise it is discarded. The strategy is executed until it does not improve the quality of the modified chromosome for a predetermined number of turns.

The result of the generation process is a set of fuzzy model rules. However, due to the iterative nature of the process, it may contain duplicated or contradictory rules. Therefore, the next step of the method is to simplify the knowledge base.

In order to perform the simplification process, the knowledge base is coded with a chromosome. Each gene of the chromosome corresponds to the occurrence of each inference rules. So it is binary encoding. An initial pool of chromosomes is generated, the first of which encodes the knowledge base obtained as a result of the generation process. The values of all its genes are one. The remaining chromosomes in the population are generated randomly. 
The generated population is subjected to the genetic algorithm for a specified number of generations. The step of the genetic algorithm consists of mutation and crossover using proportional selection. In the mutation process the mutation operator is used which changes the value at the selected gene from 1 to 0 and from 0 to 1 . In crossover process, a two-point straight crossover operator is used, which divides the parent chromosomes into three segments and replaces one of them [11]. The genetic process aims to minimize the quality function calculated according to the expressions [12] (34) - (36):

$$
Q\left(C_{j}\right)= \begin{cases}M S E\left(C_{j}\right) & \Leftrightarrow \operatorname{CL}\left(\mathrm{C}_{\mathrm{j}}\right) \geq \tau_{s F M} \\ \frac{1}{2} \cdot \sum_{r m v \in S r m v} \Delta x_{l}(r m v)^{2} & \Leftrightarrow \mathrm{CL}\left(\mathrm{C}_{\mathrm{j}}\right)<\tau_{s F M}\end{cases}
$$

where: $C_{j}$-the chromosome of the genetic algorithm, $Q$-quality function, $r m v$ - the reduced measurement vector, $S r m v$ - the set of the reduced measurement vectors, $\Delta x_{l}$ - the change of the cardinal feature of the system, $\tau_{S F M}$ - the specified threshold value of the compliance level between rule base and the set of reduced measurement vectors, $C L$ - the compliance level between rule base and the set of reduced measurement vectors, $M S E$ - the mean square error.

$$
\begin{gathered}
C L\left(C_{j}\right)=\min \left(\sum_{i=1}^{n g r b r} C F\left(F M R_{i}, r m v_{1}\right), \ldots, \sum_{i=1}^{n g r b r} C F\left(F M R_{i}, r m v_{n r m v}\right)\right) \\
\operatorname{MSE}\left(C_{i}\right)=\frac{1}{2 \cdot n r m v} \sum_{r m v \in S r m v}\left(\Delta x_{l}(r m v)-\Delta x_{F M}(r m v)\right)^{2}
\end{gathered}
$$

where: $n g r b r$ - the amount of the generated rules of the fuzzy model, $C F$ - the coefficient of the compliance between the fuzzy model rule and the reduced measurement vector, $n r m v$ - the amount of the inputs-output samples, $F R M$ - the inference rule of the fuzzy model, $\Delta x_{F M}$ - the output value of the fuzzy model.

If there are duplicate rules in the model rule base after the simplification process, copies are deleted so that each rule appears only once in the rule base.

The final step in the process is tuning the generated rules. It is also done with the use of a genetic algorithm, but this time it is an algorithm operating on chromosomes encoded with real numbers. Each chromosome describes the entire rule base by coding in individual genes the characteristic values of the fuzzy sets contained in the individual rules, being the linguistic values of the measurement variables (37).

$$
C_{j}=C_{j}^{1} C_{j}^{2} \ldots \ldots \ldots \ldots \ldots \ldots \ldots C_{j}^{n g r b r}
$$

where: $C_{j}$ - the chromosome of the genetic algorithm, ngrbr - the amount of the generated rules of the fuzzy model, $C_{j}^{i}-i$-th part of $j$-th chromosome.

$$
\begin{aligned}
C_{j}^{i}= & \left(a\left(L_{\delta p o_{1}}\left(F M R_{i}\right)\right), b\left(L_{\delta p o_{1}}\left(F M R_{i}\right)\right), c\left(L_{\delta p o_{1}}\left(F M R_{i}\right)\right),\right. \\
& a\left(L_{\delta p o_{2}}\left(F M R_{i}\right)\right), b\left(L_{\delta p o_{2}}\left(F M R_{i}\right)\right), c\left(L_{\delta p o_{2}}\left(F M R_{i}\right)\right), \\
& \vdots \\
& a\left(L_{\delta p o_{\text {nipo }}}\left(F M R_{i}\right)\right), b\left(L_{\delta p o_{\text {nipo }}}\left(F M R_{i}\right)\right), c\left(L_{\delta p o_{\text {nipo }}}\left(F M R_{i}\right)\right), \\
& \left.a\left(L_{\Delta x_{F M}}\left(F M R_{i}\right)\right), b\left(L_{\Delta x_{F M}}\left(F M R_{i}\right)\right), c\left(L_{\Delta x_{F M}}\left(F M R_{i}\right)\right)\right)
\end{aligned}
$$

where: $L_{\delta p e_{k}}\left(F M R_{i}\right)$ - the linguistic value of $k$-th input parameter appearing in $i$-th rule, $\Delta x_{F M}$ - the output value of the fuzzy model, $a, b, c$ - the characteristic values of linguistic values of the fuzzy model input and output parameters.

The domains of individual genes are determined analogously to the generation process of the model rules (4), (5), (6). The starting pool of chromosomes is randomly generated, but the first chromosome represents the rule base obtained from the simplification process. The 
arithmetic MIN-MAX operator (23) - (27) is used as the crossover operator. In mutation process the non-uniform mutation operator (29), (30) is used. The genetic algorithm aims to minimize the value of the fitness function defined by the formula (36).

\section{Results of the procedure implementation and conclusions}

The described procedure was used to create fuzzy model of the technical state changes of OP-650k-040 pulverised coal fired boiler. Performed operational tests lasted fifteen months and consisted in collecting the operational parameters of the boiler. After the preliminary analysis of the data correctness 23 parameters were selected for further considerations.

To identify the cardinal features of the boiler TKE method were taken into consideration. TKE is a common analysis method of an operation quality of power units' devices. As a result of the completed analysis the deviations $q 3$ (the deviation of the heat consumption caused by the temperature of the reheated steam $[\mathrm{kJ} / \mathrm{kWh}]$ ), $q 4$ (the deviation of the heat consumption caused by the pressure in the secondary reheater of the reheated steam $[\mathrm{kJ} / \mathrm{kWh}]$ ), $q 5$ (the deviation of the heat consumption caused by the water injections to the reheated steam $[\mathrm{kJ} / \mathrm{kWh}]$ ) and $q 8$ (the deviation of the heat consumption caused by the reduced efficiency of the boiler $[\mathrm{kJ} / \mathrm{kWh}]$ ) were chosen as cardinal features determining the technical state of the boiler [13]. Thus the task of technical state model generation was decomposed to four fuzzy models generation which model changes of the cardinal features as a functions of operational parameters time histories.

Subsequently, according to the method proposed by the author [14] the most significant operational parameters in term of the technical state change have been selected. For deviation $q 3$ the pressure of main steam, the temperature of flue gases and the amount of injected water have been selected. For deviation $q 4$ the temperature of main steam, the content of oxygen in flue gasses before rotary air preheater and the content of oxygen in flue gasses after rotary air preheater have been selected. For deviation $q 5$ it was the temperature of reheated steam and the temperature of feed water and for deviation $q 8$ the amount of injected water.

In order to generate the models of the operational parameters time histories impact on the boiler features values, for each deviation the learning and testing data sets were prepared. Each of the sets consisted of the most significant measurement collections which included input and output data of the model. Subsequently, on the basis of learning data set the automatic generation of the models was performed according to the procedure presented in the paper.

Table 1. The values of the operation quality measures of the models.

\begin{tabular}{|c|c|c|c|c|}
\hline Measure of the operation quality & $\begin{array}{c}\text { model - } \\
\text { deviation q3 }\end{array}$ & $\begin{array}{c}\text { model - } \\
\text { deviation q4 }\end{array}$ & $\begin{array}{c}\text { model - } \\
\text { deviation q5 }\end{array}$ & $\begin{array}{c}\text { model - } \\
\text { deviation q8 }\end{array}$ \\
\hline Relative minimum error [\%] & 0.2498 & 1.2772 & 1.2606 & 1.3084 \\
\hline Relative maximum error [\%] & 155.3265 & 92.9864 & 92.0827 & 84.574 \\
\hline Relative mean error [\%] & 13.8125 & 5.129 & 9.4532 & 11.4643 \\
\hline Relative mean square error [\%] & 1.1368 & 0.6205 & 0.8314 & 0.9089 \\
\hline Correlation function value [\%] & 63.2596 & 60.7267 & 60.7023 & 82.1394 \\
\hline
\end{tabular}


Finally, the verification of the generated fuzzy models was performed. To do it, for each model the relative minimum error, the relative maximum error, the relative mean error, the relative mean square error and the value of the correlation function were determined (Table 1). The values of the measures were determined taking into consideration the values calculated using the real collected data and the values calculated by the models for testing data set.

Analysing the calculated values of the operation quality measures of the models of the operational parameters time histories influence on the values of the system features it was decided that the proposed method of the models generation is efficient enough to apply it to the conducted research.

\section{References}

1. O. Cordon, Genetic fuzzy systems: evolutionary tuning and learning of fuzzy knowledge bases (World Scientific, 2001)

2. F. Herrera, M. Lozano, J.L. Verdegay, Generating fuzzy rules from examples using genetic algorithms, Fuzzy Logic and Soft Computing, pp. 11-20 (1995)

3. F. Herrera, M. Lozano, J.L. Verdegay, A learning process for fuzzy control rules using genetic algorithms, Fuzzy Set Syst, Vol. 100 Issue 1-3, pp. 143-158 (1998)

4. O. Cordon, F. Herrera, L. Sanchez, Evolutionary Learning Processes for Data Analysis in Electrical Engineering Applications (John Wiley \& Sons Ltd. 1997)

5. O. Cordon, M.J. del Jesus, F. Herrera, M. Lozano, MOGUL: A methodology to obtain genetic fuzzy rule-based systems under the iterative rule learning approach, Int J Intell Syst, Vol. 14 Issue 11, pp. 1123-1153 (1999)

6. A. Gonzblez, P. Raúl, SLAVE: A genetic learning system based on an iterative approach, IEEE T Fuzzy Syst, Vol. 7 Issue 2, pp. 176-191 (1999).

7. O. Cordon, F. Herrera, Hybridizing genetic algorithms with sharing scheme and evolution strategies for designing approximate fuzzy rule-based systems, Fuzzy Set Syst, Vol. 118 Issue 2, pp. 235-255 (2001)

8. M. Pająk, Fuzzy modelling of temperature difference in $200 \mathrm{MW}$ power unit condenser using genetic fuzzy systems, Control Cybern, Vol. 37 Issue 3, pp. 565-583 (2008)

9. M. Pająk, Genetic - Fuzzy system of power units maintenance schedules generation, Journal of Intelligent and Fuzzy Systems, Vol. 28 Issue 4, pp. 1577-1589 (2015)

10. T. Bäck, Evolutionary Algorithms in Theory and Practice: Evolution Strategies, Evolutionary Programming, Genetic Algorithms (Oxford Scholarship Online, 2020)

11. Padmavathi Kora, Priyanka Yadlapalli, Crossover Operators in Genetic Algorithms: A Review, Int J Comput Appl, Vol. 162 Issue 10, pp. $34-36$ (2017)

12. A. González, F. Herrera, Multi-stage genetic fuzzy systems based on the iterative rule learning approach, Mathware \& Soft Computing, Vol. 4 Issue 3, pp. 233-249 (1997)

13. Ł. Muślewski, M. Pająk, B. Landowski, B. Żółtowski, A method for determining the usability potential of ship steam boilers, Pol Marit Res, Vol. 92 Issue 4, pp. 105-112 (2016)

14. M. Pająk, Identification of the operating parameters of a complex technical system important from the operational potential point of view, P I Mech Eng I-J Sys, Vol. 232 Issue 1, pp. 62-78 (2018) 